\title{
Lamb Wave Directional Sensing with Piezoelectric Fiber Rosette in Structure Health Monitoring
}

\author{
Songlai Wang, ${ }^{1}$ Wanrong Wu $\mathbb{D}^{1},{ }^{1}$ Yiping Shen $\mathbb{D}^{2}{ }^{2} \mathrm{Hui} \mathrm{Li}^{2}$ and Binlong Tang ${ }^{2}$ \\ ${ }^{1}$ College of Mechanical and Electrical Engineering, Central South University, Changsha 410083, China \\ ${ }^{2}$ Hunan Provincial Key Laboratory of Health Maintenance for Mechanical Equipment, \\ Hunan University of Science and Technology, Xiangtan 411201, China \\ Correspondence should be addressed to Yiping Shen; yiping1011@163.com
}

Received 25 February 2019; Revised 13 May 2019; Accepted 2 June 2019; Published 24 June 2019

Academic Editor: Adam Glowacz

Copyright (c) 2019 Songlai Wang et al. This is an open access article distributed under the Creative Commons Attribution License, which permits unrestricted use, distribution, and reproduction in any medium, provided the original work is properly cited.

Directional piezoelectric sensors can detect the Lamb wave propagation direction to locate damage in structural health monitoring (SHM). The directivity of the round piezoelectric fiber is exploited with a $0^{\circ} / 45^{\circ} / 90^{\circ}$ rosette configuration to acquire flexural Lamb wave signals. The directional response of the piezoelectric fiber under narrowband tone-burst excitation is theoretically deduced. Experimental tests are conducted to demonstrate the directivity and the frequency response property of the piezoelectric fiber under different excitation central frequencies in comparison with the MFC, rectangular piezoelectric sheet, and circular piezoelectric disc. Continuous wavelet transform (CWT) is applied to extract the maximum response amplitude information of the acquired Lamb wave signal at a central frequency. Experimental test results indicate that the piezoelectric fiber is capable to be used as a Lamb wave directional sensor than other piezoelectric sensors. A numerical estimation method for the Lamb wave propagation direction is proposed by defining an error function between the theoretical and experimental normalized response amplitude. The proposed method is generally applicable for different rosette configurations. Experimental results validate the accuracy of the proposed estimation method. The research results are significant to design or select the piezoelectric sensor to measure Lamb wave signals.

\section{Introduction}

Structural health monitoring (SHM) is an emerging technique that is capable of detecting damage and evaluating structural integrity and reliability. Lamb wave-based damage detection in plate-like structures draws more and more attention because the Lamb wave can travel over a long distance even in material with high attenuation and be highly susceptible to small damage on a propagation path [1-4]. Piezoelectric sensors made of lead zirconate titanate (PZT) ceramics are widely used in SHM applications. However, the brittle nature of monolithic piezoceramic restricts their applications in many areas, especially in curved or irregularly shaped structures, such as the wind turbine blade $[5,6]$ and aircraft and aerospace composite structures [7-10]. Recently, various piezoelectric fiber composites (PFCs) have been developed to overcome such most serious drawbacks, such as active fiber composites (AFCs) [11, 12] and macrofiber composite (MFC) [8, 13]. The directional sensitivity of piezoelectric composite sensors makes them more attractive to detect the Lamb wave propagation direction $[2,8,9,14]$. The direction information is significant to improve the SHM strategy in comparison with the traditional ToF-based approach $[8,15]$. Theoretically, the Lamb wave source location can be simply determined by intersecting the wave directions detected by two such sensors.

Piezoelectric sensors with preferable directional sensitivities are the most suitable sensors to locate damage for SHM applications. Kawiecki and Jesse [16] demonstrated experimentally that the piezoelectric rosette, similar to the strain gage rosette, can produce more damage-related information to improve the damage detection performance. Lanza Di Scalea et al. [17] and Matt and Scalea [18] 
exploited the directional sensitivities of the rectangular piezoelectric sheet and MFC and used a delta rosette configuration for acoustic emission (AE) localization. Salamone et al. [19] conducted a impact location test with MFC rosettes in a high-velocity regime on both isotropic and anisotropic plates. Kijanka et al. [20] uses two MFC rosettes to locate damage in an aluminum plate under temperature variation. Collet et al. [21] focused on the directivity properties when MFC actuated individually, or as part of rosette configuration, and compared with the monolithic circular piezoelectric disc and rectangular piezoelectric sheet. Considering the anisotropic material of MFC, the direction sensitivity is decomposed into longitudinal and transverse sensitivity factors [18]. The directional sensitivity of the piezoelectric sheet is same with MFC except for the isotropic properties [17].

As for the other PFCs, Mańka et al. [22] investigated three different transducers made of PFCs to find which one has the best directivity for SHM applications. Lin et al. [9] proposed a PFC sensor consisting of unidirectionally aligned rectangular piezoelectric fibers and investigated its sensing and localization performance using the $\mathrm{AE}$ technique compared with the MFC and piezoelectric disk. Liu et al. [23] investigated the high directivity of the metal-core piezoelectric fiber (MPF), and a new imaging method with the MPF rosette is proposed to improve the accuracy of impact localization [24]. Compared with the piezoelectric sheet and MFC, MPF has higher directivity because MPF's diameter is smaller than the width of MFC, and the strain component in the transverse sensor direction can be ignored [24].

In this paper, three round piezoelectric fibers with a $0 \%$ $45^{\circ} / 90^{\circ}$ rosette configuration are applied for Lamb wave propagation direction estimation. The directional response of the piezoelectric fiber to the flexural Lamb wave is investigated by theoretical modeling under narrowband toneburst excitation. Continuous wavelet transform (CWT) is applied to extract the maximum response amplitude information of the acquired Lamb wave signal at a central frequency. Theoretical and experimental results demonstrate the directivity and response sensitivity of the piezoelectric fiber in comparison with various piezoelectric sensors. A numerical estimation method is proposed to determine the Lamb wave propagation direction. Experimental tests with different frequencies are conducted to validate the effectiveness of the proposed method.

\section{Directional Response Sensoring with the Piezoelectric Fiber Rosette}

2.1. Material and Procedures of the Piezoelectric Fiber Rosette. Piezoelectric fiber rosette consisted of three round piezoelectric fibers with a $0^{\circ} / 45^{\circ} / 90^{\circ}$ rosette configuration, as shown in Figure 1(a). The piezoelectric fiber with a length of $10 \mathrm{~mm}$ is cut from an original piezoelectric fiber with the length of $150 \mathrm{~mm}$ and the diameter of $0.8 \mathrm{~mm}$, produced by Smart Material Corp. The piezoelectric material is PZT SP505 (Navy type II). The electrodes at two ends of a piezoelectric fiber are covered with silver paint with $10 \mathrm{~min}$ curing at a room temperature. The polarization of the piezoelectric fiber is conducted in silicone oil with a constant DC voltage of $20 \mathrm{kV}$ for $10 \mathrm{~min}$. The homemade polarization device is shown in Figure 1(b). Three piezoelectric fibers have similar piezoelectric constants $d_{33}$ around $370 \mathrm{pC} / \mathrm{N}$ measured by a ZJ-6A $d_{33}$ piezometer system provided by Institute of Acoustics, Chinese Academy of Sciences.

The fabrication process of the piezoelectric fiber rosette, similar with the MPF rosette, combines the flexible printed circuit technique with the PFC manufacturing process. Polyimide film with a printed circuit is used as the sensor matrix. Three piezoelectric fibers are directly placed into the printed circuit on the polyimide matrix with conductive adhesive at two respective electrodes. Six signal wires are firmly wired to the output of the printed circuit and connected to the signal collector. Since the single piezoelectric fiber is very brittle, the piezoelectric fiber rosette assembly is covered with an epoxy resin to protect them from breakdown in practical use. Therefore, the piezoelectric fiber rosette can be applied to curved or irregularly shaped structures.

\subsection{Directional Response of the Piezoelectric Fiber to Flexural} Lamb Wave. Assume that piezoelectric fibers are well bonded to the top surface of a plate with a thickness of $2 h$, as shown in Figure 2. Flexural Lamb wave, namely, $\mathrm{A}_{0}$ mode motion, is dominated in low frequency, while the $S_{0}$ mode is dominated beyond $100 \mathrm{kHz}[25,26]$. The flexural Lamb wave is commonly used for damage detection as its short wavelength and low wave velocity. Therefore, the flexural Lamb wave in the low-frequency range is mainly focused in this paper. The angle between the wave propagation direction $x^{\prime}$ and the lengthwise direction of the piezoelectric fiber is defined as $\theta$. The origin of the global coordinate system is in the midplane of the plate structure. Consider the constitutive relations for a piezoelectric material, and the generated charge is expressed as follows [27]:

$$
Q=\frac{1}{l} \int_{-(l / 2)}^{l / 2} E d_{33} \varepsilon_{Z Z} \pi r^{2} d Z=\frac{E d_{33} \pi r^{2}}{l} \int_{-(l / 2)}^{l / 2} \varepsilon_{Z Z} d Z,
$$

where $E$ is Young's modulus of the piezoelectric fiber, $d_{33}$ is the piezoelectric constant, $\varepsilon_{z z}$ is the strain component in the lengthwise direction of the piezoelectric fiber, and $r$ and $l$ are the radius and the length.

Piezoelectric fiber can be considered as a capacitor, and its capacitance is written as

$$
C_{\mathrm{p}}=\frac{e_{33} \pi r^{2}}{l}
$$

where $e_{33}$ is the dielectric permittivity.

The voltage generated across the piezoelectric fiber electrode is expressed as

$$
V=\frac{Q}{C_{\mathrm{p}}}=\frac{E d_{33}}{e_{33}} \int_{-(l / 2)}^{l / 2} \varepsilon_{Z Z} d Z .
$$

As presented in [18], the in-plate displacement of the flexural Lamb wave can be written as 


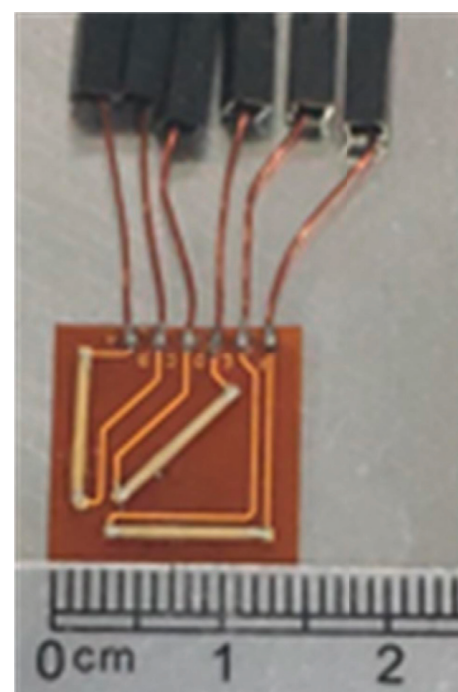

(a)

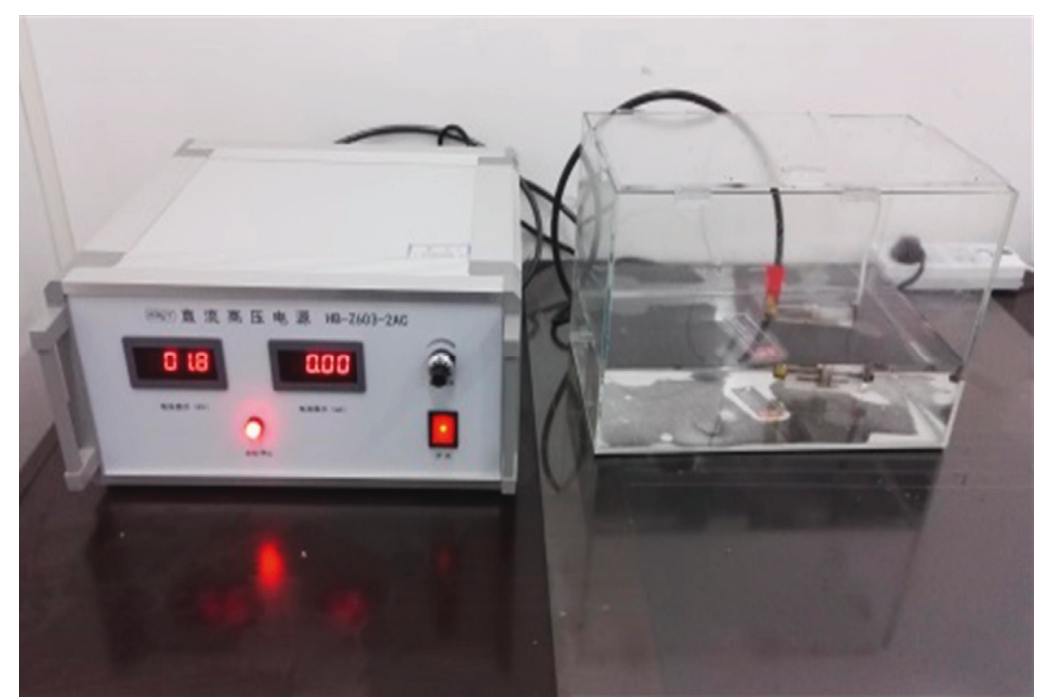

(b)

FIguRe 1: (a) Piezoelectric fiber rosette; (b) the homemade polarization device for the piezoelectric fiber.

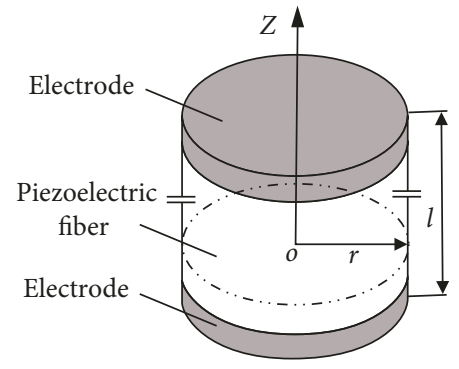

(a)

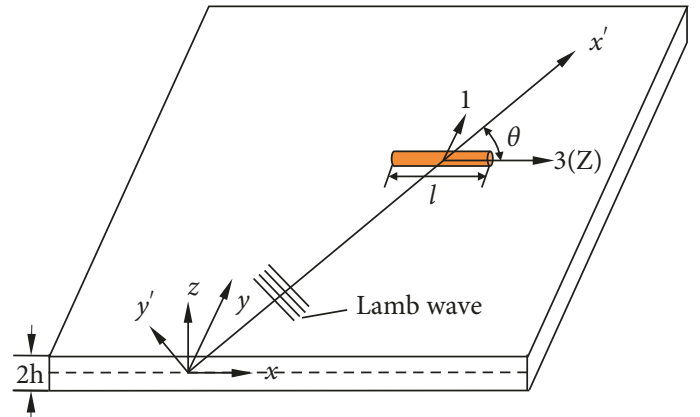

(b)

Figure 2: Piezoelectric fiber sensing analysis in local and global coordinate systems: (a) local coordinate system; (b) global coordinate system.

$$
u_{x^{\prime}}=B k\left(\frac{\sinh a z}{\cosh a h}-\frac{2 a b}{k^{2}+b^{2}} \cdot \frac{\sinh b z}{\cosh b h}\right) e^{i\left(k x^{\prime}-\omega t-(\pi / 2)\right)},
$$

where $B$ is an arbitrary constant, and the parameters $a$ and $b$ are defined as

$$
\begin{aligned}
& a=\sqrt{k^{2}-\frac{\omega^{2}}{c_{\mathrm{L}}^{2}}}, \\
& b=\sqrt{k^{2}-\frac{\omega^{2}}{c_{\mathrm{T}}^{2}}}, \\
& k=\frac{2 \pi}{\lambda},
\end{aligned}
$$

where $k, \lambda$, and $\omega$ are the wavenumber, wavelength, and the circular frequency and $c_{\mathrm{L}}$ and $c_{\mathrm{T}}$ are the longitudinal and transversal velocity in the plate, respectively.

For the plane wave $\left(\varepsilon_{y^{\prime} y^{\prime}}=0\right)$, the in-plane strain in the wave propagation direction is expressed as

$$
\begin{aligned}
\left.\varepsilon_{x^{\prime} x^{\prime}}\right|_{z=h} & =\left.\frac{\partial u_{x^{\prime}}}{\partial x^{\prime}}\right|_{z=h} \\
& =i k^{2} B\left(\tanh a h-\frac{2 a b}{k^{2}+b^{2}} \tanh b h\right) e^{i\left(k x^{\prime}-\omega t-(\pi / 2)\right)} .
\end{aligned}
$$

The strain $\varepsilon_{x^{\prime} x^{\prime}}$ can be decomposed into the lengthwise direction of the piezoelectric fiber:

$$
\varepsilon_{Z Z}=\varepsilon_{x^{\prime} x^{\prime}} \cos ^{2} \theta
$$

Substituting equation (7) into equation (3) and employing the coordinate transformation $x^{\prime}=x \cos \theta+$ $y \sin \theta$ and Euler's formulate $e^{i a}-e^{-i a}=2 i \sin a$, the piezoelectric fiber's response to the flexural Lamb wave is as follows $[17,18]$ :

$$
V=i \bar{V} e^{-i(\omega t+(\pi / 2))}
$$

where the amplitude is 


$$
\bar{V}=\frac{N k E d_{33}}{e_{33}} \cos \theta \sin \left(\frac{l k \cos \theta}{2}\right) .
$$

The constant $N$ is

$$
N=2 B\left(\tanh a h-\frac{2 a b}{k^{2}+b^{2}} \tanh b h\right) .
$$

According to equation (6) and $k=2 \pi / \lambda$, equation (9) can be rewritten as

$$
\bar{V}=S \bar{\varepsilon}_{x^{\prime} x^{\prime}}=\frac{E d_{33} \lambda}{\pi e_{33}} \cos \theta \sin \left(\frac{\pi l \cos \theta}{\lambda}\right) \bar{\varepsilon}_{x^{\prime} x^{\prime}},
$$

where $\bar{\varepsilon}_{x^{\prime} x^{\prime}}$ is the amplitude of the in-plane strain under excitation. $S$ is the sensitivity factor, defined as

$$
S=\frac{E d_{33} \lambda}{\pi e_{33}} \cos \theta \sin \left(\frac{\pi l \cos \theta}{\lambda}\right)
$$

Harmonic flexural Lamb wave excitation is assumed in equation (11). However, narrowband tone-burst signals are typically used to excite the Lamb wave in SHM applications. As for a linear system, the response amplitude $\bar{U}(\omega)$ to a narrowband excitation can be calculated from the product of the frequency-domain response to harmonic excitation $\bar{V}(\omega)$ and the amplitude of the Fourier transform (FT) of the excitation. FT amplitude of the narrow tone burst of central frequency $\omega_{c}$ can be expressed as follows [17]:

$$
A(\omega)=A_{\mathrm{e}}\left(\frac{\sin n \pi\left(\omega / \omega_{\mathrm{c}}+1\right)}{\omega+\omega_{\mathrm{c}}}-\frac{\sin n \pi\left(\omega / \omega_{\mathrm{c}}-1\right)}{\omega-\omega_{\mathrm{c}}}\right),
$$

where $A_{\mathrm{e}}$ is the excitation amplitude and $n$ is the cycle number of the tune-burst excitation signal.

The response amplitude to narrowband tone-burst excitation can be expressed as

$$
\begin{aligned}
\bar{U}(\omega)=S A(\omega)= & A_{\mathrm{e}}\left(\frac{\sin n \pi\left(\omega / \omega_{\mathrm{c}}+1\right)}{\omega+\omega_{\mathrm{c}}}-\frac{\sin n \pi\left(\omega / \omega_{\mathrm{c}}-1\right)}{\omega-\omega_{\mathrm{c}}}\right) \\
& \cdot \frac{E d_{33} \lambda}{\pi e_{33}} \cos \theta \sin \left(\frac{\pi l \cos \theta}{\lambda}\right) .
\end{aligned}
$$

It is clear that the response depends on the central circular frequency $\omega_{c}$, the angle $\theta$, and the wavelength tuning effect in term $\sin (\pi l \cos \theta / \lambda)$. The largest amplitude is at central frequency $\omega_{c}$ when $\lim _{\omega \longrightarrow \omega_{c}} \sin n \pi\left(\omega / \omega_{c}-1\right) /$ $\left(\omega-\omega_{c}\right)=n \pi / \omega_{c}$. The largest amplitude is expressed as follows:

$$
\bar{U}_{\max }=A_{\mathrm{e}} \frac{n E d_{33} \lambda^{2}}{2 \pi e_{33}} \cos \theta \sin \left(\frac{\pi l \cos \theta}{\lambda}\right) .
$$

More detailed response equation deductions have been presented in [17]. Compared with harmonic excitation, the maximum amplitude of voltage response is only scaled-up with a constant. Similarly to what was investigated by Giurgiutiu [25] and Lanza Di Scalea et al. [17], wavelength tuning effects emerge through terms $\sin (\pi l \cos \theta / \lambda)$, and the response of the piezoelectric fiber in $0^{\circ}$ will be zero at $\lambda=l / n$ for $n=1,2,3 \ldots$.

Under same excitation motion, the directional response can be expressed as an angle-related function:

$$
f(\theta)=\cos \theta \sin \left(\frac{\pi l \cos \theta}{\lambda}\right) .
$$

When the piezoelectric fiber is parallel with the Lamb wave propagation direction, equation (16) is rewritten as follows:

$$
f(\theta)_{\theta=0}=\sin \left(\frac{\pi l}{\lambda}\right) .
$$

It is found that the piezoelectric fiber shows same directivity with MPF, which with no transverse effects due to its small diameter compared with rectangular MFC and piezoelectric sheet $[17,18,24]$. These response equations and the angle-related functions are presented in equations (A.1)-(C.2) in Appendix. The $d_{33}$ effect is dominated in the piezoelectric fiber, whereas the $d_{31}$ effect is dominated in MFC. MPF can overcome the brittleness of monolithic piezoceramic because of the metal core, but it is not commercially available. The directivity sensitivity will be demonstrated by an experimental test in comparison with various piezoelectric sensors under different excitation frequencies in Section 3.

2.3. Lamb Wave Propagation Direction Estimation Method. The directional sensitivity characteristics made the piezoelectric fiber suitable for detecting the Lamb wave propagation direction. Since the Lamb wave propagation direction is in accordance with the principle strain direction, Betz et al. [15] and Matt and Scalea [18] derived the principal strain angle equations by strain transformation related with the measured signal of the rosette. Zhang et al. [24] presented an imaging method with an numerical computation to estimate the approximate Lamb wave propagation direction angle. The proposed Lamb wave propagation angle estimation method is an extension of the Zhang and Qiu's numerical method [24], which is capable to treat various rosette configurations.

Assume Lamb wave propagation angle is $\theta$ with the piezoelectric fiber in $0^{\circ}$, as shown in Figure 3. Considering the rosette configuration angle $\alpha_{i}$ and the response amplitude equation in equation (15), the voltage response amplitude of three piezoelectric fibers can be expressed as follows:

$$
\begin{aligned}
& \bar{U}_{i \max }=\bar{U}_{\max } \cos \left(\theta+\alpha_{i}\right) \sin \left(\frac{\pi l \cos \left(\theta+\alpha_{i}\right)}{\lambda}\right), \\
& \alpha_{i}=0^{\circ}, 45^{\circ}, \text { and } 90^{\circ},
\end{aligned}
$$

where $\bar{U}_{\max }$ is the maximum voltage response of the piezoelectric fiber which is located in parallel with the Lamb wave propagation direction. 


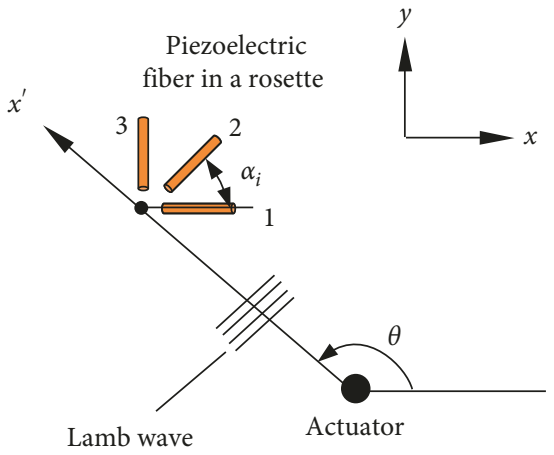

Figure 3: Estimation of the Lamb wave propagation direction.

The directivity of the rosette sensor is approximated by a cosine-squared function in $[15,24], f(\theta) \approx \cos ^{2} \theta$. Such approximation is only reasonable in the low-frequency range around $15 \mathrm{kHz}$. As for the $120^{\circ}$ rosette configuration, the sum of the response amplitudes of three MPFs is a constant, which can be easily deduced based on the trigonometric equations, and is used to normalize the response of each MPF. As for the common $0^{\circ} / 45^{\circ} / 90^{\circ}$ rosette configuration of the piezoelectric fiber, the sum is not a constant by using equation (18). More generally, the sum is still used to normalize the response voltage. Therefore, the normalized amplitudes of piezoelectric fibers can be expressed as

$$
\begin{array}{r}
T_{i}=\frac{3 \bar{U}_{i \max }}{\sum_{i=1}^{3} \bar{U}_{i \max }}=\frac{3 \cos \left(\theta+\alpha_{i}\right) \sin \left(\pi l \cos \left(\theta+\alpha_{i}\right) / \lambda\right)}{\sum_{i=1}^{3} \cos \left(\theta+\alpha_{i}\right) \sin \left(\pi l \cos \left(\theta+\alpha_{i}\right) / \lambda\right)}, \\
\alpha_{i}=0^{\circ}, 45^{\circ} \text {, and } 90^{\circ} .
\end{array}
$$

It can be found that the normalized amplitudes $T_{i}$ are only related to the angle $\theta$. The Lamb wave direction angle can be calculated by the voltage responses of three piezoelectric fibers in a rosette. The angle $\theta$ can be evaluated by the error between the experimental and the theoretical normalized voltage amplitude using the numerical computation method. The error is defined as

$$
e(\hat{\theta})=\sqrt{\frac{1}{3} \sum_{i=1}^{3}\left(T_{i}-T_{i}(\hat{\theta})\right)^{2}},
$$

where $\hat{\theta}$ is the estimation of the Lamb wave propagation direction for theoretical calculation in equation (19).

According to equation (20), the error value $e(\hat{\theta})$ will be 0 when $\hat{\theta}=\theta$. In practical application, $e(\hat{\theta})$ is impossible to be 0 because of the unavoidable measurement error. Therefore, the estimation of $\theta$ is assumed to be $\hat{\theta}$ when $e(\hat{\theta})$ trends to the minimum. The developed method is applicable to the $120^{\circ}$ rosette configuration without the cosine-square approximation equation. The significant advantage of the proposed method is the averaging errors of three piezoelectric fibers in a rosette compared with the analytical principal strain equation. No absolute signal values are needed by using the normalized response amplitudes. As for the damage detection, no arrival time is needed, which is difficult to be measured because of the dispersion characteristics of the Lamb wave.

\section{Theoretical Analysis and Experimental Results and Discussion}

3.1. Experimental Setup. Three experimental tests are performed to demonstrate the capability of the piezoelectric fiber rosette as a directional strain sensor for Lamb wave propagation direction estimation. The first is the directivity test of the piezoelectric fiber under different excitation frequencies, the second is the frequency response test of the piezoelectric fiber at different arranged angles, and the third is the Lamb wave propagation direction test with two piezoelectric fiber rosettes. Various piezoelectric sensors, e.g., MFC, rectangular piezoelectric sheet, and circular piezoelectric disc, are used in comparison with the piezoelectric fiber. The dimension of MFC M2814-P2 is $28 \mathrm{~mm} \times 14 \mathrm{~mm} \times 0.3 \mathrm{~mm}$. Piezoelectric sheet and piezoelectric disc are produced by Jiangsu Paizhou Electronic Technology Company. The dimension of the piezoelectric sheet is $20 \mathrm{~mm} \times 10 \mathrm{~mm} \times 0.6 \mathrm{~mm}$. The radius of the piezoelectric disc is $10 \mathrm{~mm}$, and the thickness is $0.3 \mathrm{~mm}$.

Experimental tests are conducted on a $1 \mathrm{~m} \times 1 \mathrm{~m} \times$ $1.5 \mathrm{~mm}$ aluminum plate. $c_{\mathrm{L}}$ and $c_{\mathrm{T}}$ are $6240 \mathrm{~m} / \mathrm{s}$ and $3040 \mathrm{~m} /$ $\mathrm{s}$, respectively, and the phase and group velocities are plotted in Figure 4. Narrowband tone-burst signals are used to excite the Lamb wave. Tone-burst signals are simply sine waves modulated by Hamming window. A 5-cycle tone-burst signals at a central frequency of $80 \mathrm{kHz}$ is shown in Figure 5(a), and its FFT is shown in Figure 5(b). These experiments herein are conducted using the piezoelectric disc to excite the Lamb wave in the plate.

EPA-10 power amplifier, produced by the Piezo System Inc. (USA), is used to amplify the excitation narrowband signals. The $80 \mathrm{~V}$ peak-to-peak amplification excitation is applied to the actuator. NI PXle-6361 platform is applied to collect the response output voltage of piezoelectric sensors. Various piezoelectric sensors are directly connected to the platform to acquire the electric signals generated through the piezoelectric coupling between strain field and electric field. Experimental setup is shown in Figure 6(a).

As for the directivity test, seven actuators of piezoelectric discs are placed evenly in the quarter circular at angle intervals of $15^{\circ}$, as shown in Figure 6(b). The distance between the actuators and the sensors is $200 \mathrm{~mm}$. The sampling frequency is $2 \mathrm{MS} / \mathrm{s}$. The measured signals of the piezoelectric sensors are averaged 100 times. A National Instruments LabVIEW program was written to generate the excitation wave and acquire the sensor data, and Matlab program was written for Lamb wave signal analysis, as well as Lamb wave propagation computation.

A voltage response of the piezoelectric fiber in $0^{\circ}$ around central frequency of $80 \mathrm{kHz}$ is shown in Figure $7(\mathrm{a})$. Because the acquired signals of piezoelectric fibers are so 


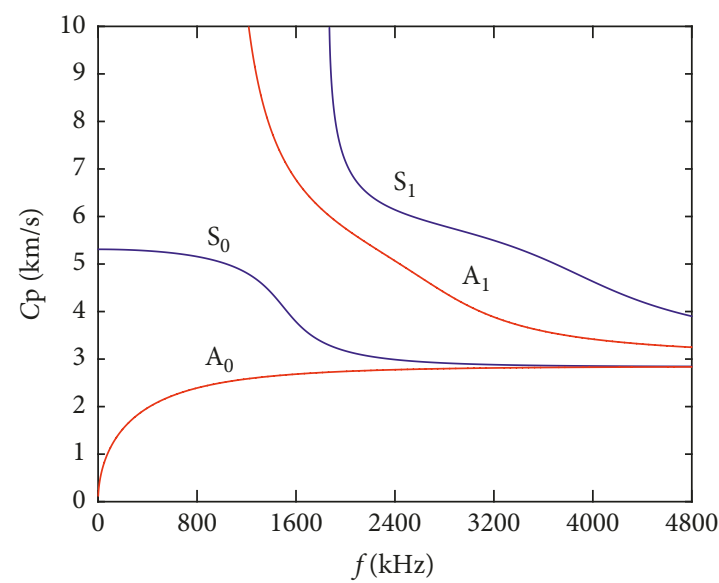

(a)

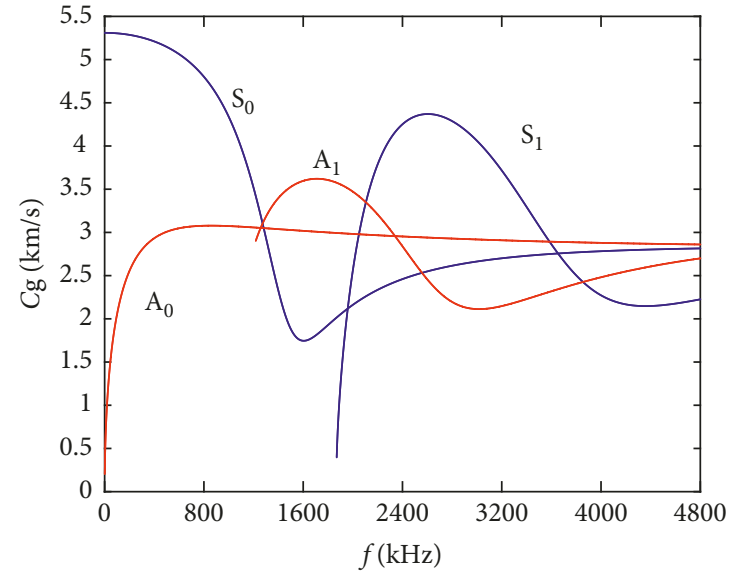

(b)

Figure 4: Disperse curve of (a) phase velocity and (b) group velocity.

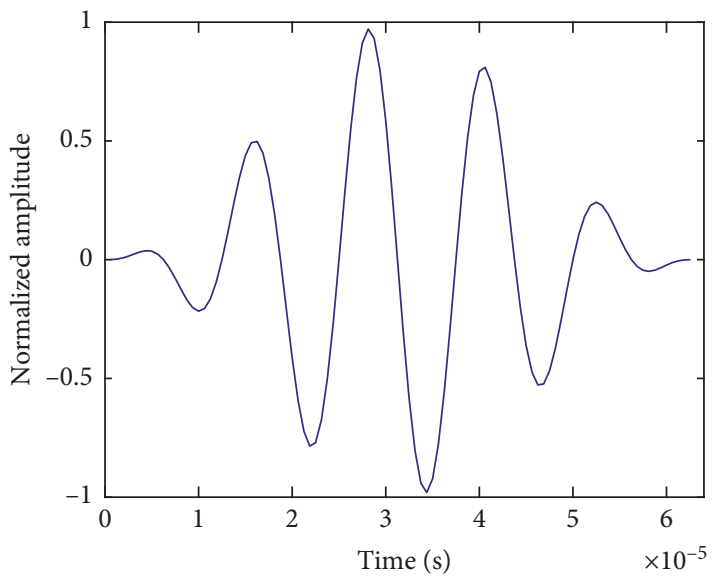

(a)

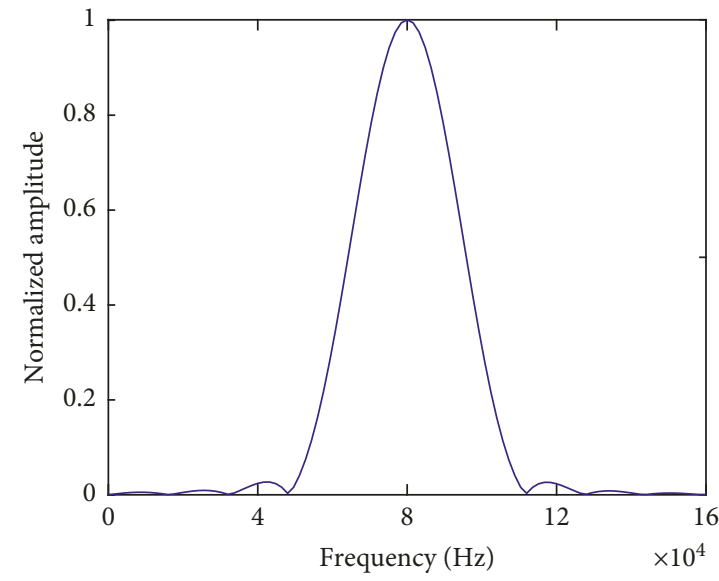

(b)

FIgURe 5: Narrowband tone-burst excitation: (a) 5-cycle tone burst at a central frequency $80 \mathrm{kHz}$; (b) FFT of the excitation wave.

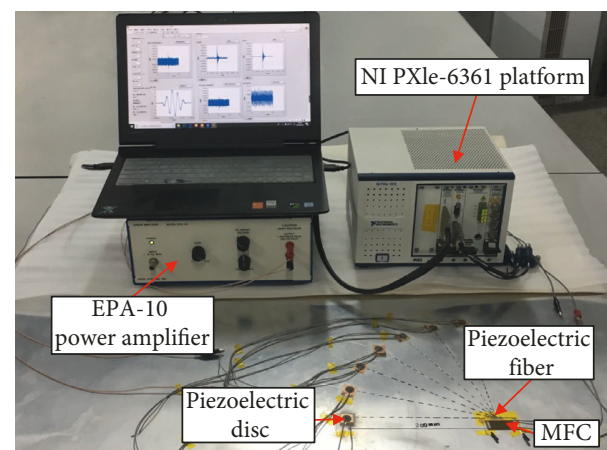

(a)

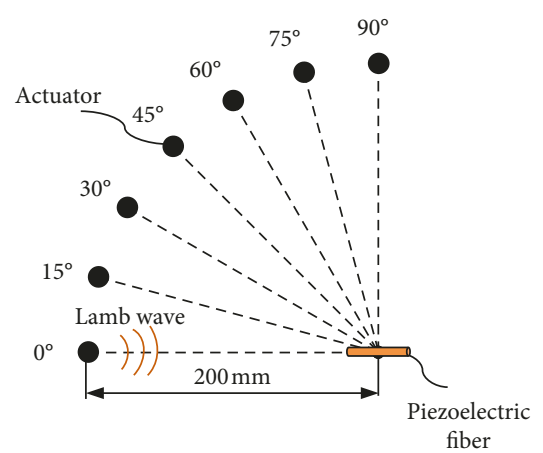

(b)

FIgURE 6: (a) Experimental test setup; (b) configuration of actuators.

weak to be submerged with the noise, it is a tricky work to extract the maximum response amplitude to narrowband excitation at a certain central frequency. As for the Lamb wave propagation direction estimation in this paper, the first arrival flexural Lamb waves are used to extract the amplitudes at central frequencies. The general practice in signal processing is to utilize time-frequency representation [28]. Wavelet transform (WT) has gained popularity as 


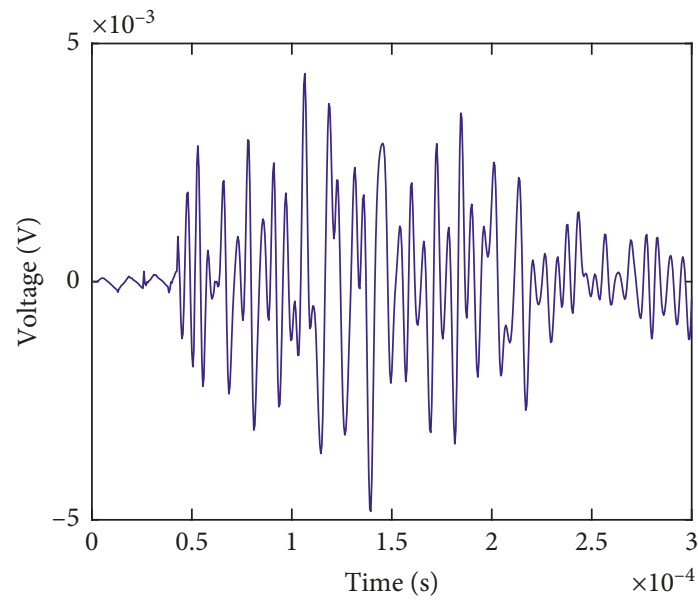

(a)

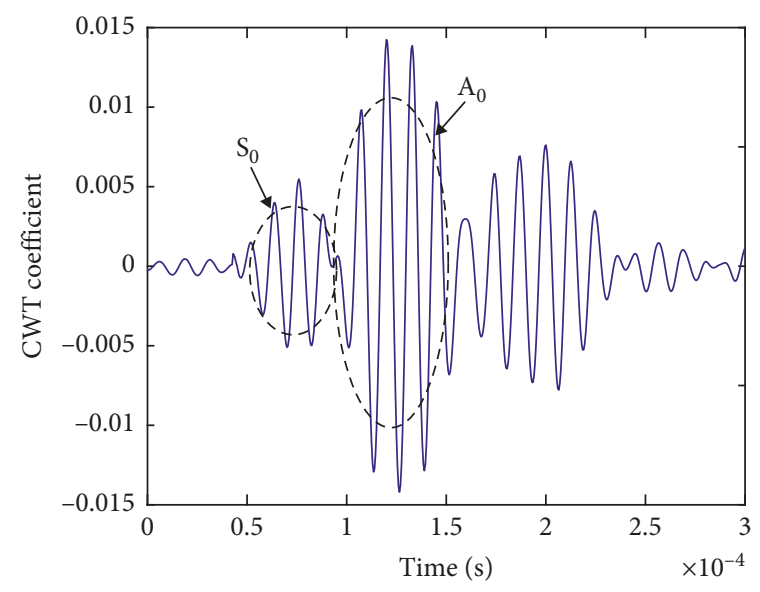

(b)

Figure 7: Lamb wave signal acquired by the piezoelectric fiber at $80 \mathrm{kHz}$ : (a) averaged after 100 times; (b) CWT coefficients at $80 \mathrm{kHz}$.

an efficient signal processing to extract signal features from Lamb wave signals. Continuous wavelet transform (CWT) is particularly effective for signal decomposition and feature extraction [1]. Essentially, the decomposition of CWT is implemented by finding a signal correlation with the mother wavelet $\psi(t)$ :

$$
\operatorname{CWT}\left(a_{\mathrm{c}}, b_{\mathrm{c}}\right)=\frac{1}{\sqrt{a_{\mathrm{c}}}} \int_{-\infty}^{+\infty} U(t) \psi^{*}\left(\frac{t-b_{\mathrm{c}}}{a_{\mathrm{c}}}\right) d t,
$$

where $U(t)$ is the measured voltage of the piezoelectric fiber and $\psi^{*}(t)$ is the conjugate of the mother wavelet $\psi(t)$ with the scale parameter $a_{\mathrm{c}}$ and the position parameter $b_{c}$.

The mother wavelet $\psi(t)$ determines the accuracy and efficiency of the transformation. Since the excitation signal is a 5-cycle ton-burst signal, the Morlet wavelet is well capable to extract the amplitude information at a certain frequency from the complex Lamb wave signal $[29,30]$. The coefficients of CWT of the piezoelectric fiber in $0^{\circ}$ at a central frequency of $80 \mathrm{kHz}$ is plotted in Figure 7(b). The maximum coefficient of CWT at a certain central frequency of the first arrival flexural Lamb wave is used to represent the maximum amplitude of the piezoelectric fiber.

3.2. Directivity Test Results. The directivity test is conducted to validate the capability of the piezoelectric fiber to acquire Lamb wave signals with related-direction information. According to equation (16), the directivity in theoretical response is plotted in Figure $8(\mathrm{a})$. It can be found that the voltage amplitude of the piezoelectric fiber is strongly dependent on the angle $\theta$. The amplitude is decreasing as $\theta$ increased from $0^{\circ}$ to $90^{\circ}$ in the low-frequency range below $80 \mathrm{kHz}$. The response voltage is largest at $0^{\circ}$ and smallest at $90^{\circ}$. The voltage amplitude is tuned beyond the excitation frequency of $80 \mathrm{kHz}$. The reason is that the length of the piezoelectric fiber is comparable with the wavelength; e.g., the wavelength is $11.38 \mathrm{~mm}$ at frequency of $100 \mathrm{kHz}$, as listed in Table 1 . The experimental directivity at $20 \mathrm{kHz}$ is shown in Figure 8(b), which is in well agreement with the theoretical curve. Experimental directivities are plotted in Figure 8(c) in the low-frequency range from $20 \mathrm{kHz}$ to $80 \mathrm{kHz}$, where the response amplitudes are normalized by the maximum response amplitudes of the piezoelectric fiber in $0^{\circ}$ at a central frequency $20 \mathrm{kHz}$. It can be seen that the experimental directivity curves are in well agreement with the theoretical directivity curves. Compared to the response amplitudes of the piezoelectric fiber in $0^{\circ}$ at different frequencies, the voltage amplitude is decreasing as the excitation frequency increasing. The frequency response properties of the piezoelectric fiber account for such decreases, which are determined by the impedance characteristics of the piezoelectric fiber. Consequently, the response properties under different excitation central frequencies are investigated in Section 3.3.

According to equations (A.2) to (B.2) in Appendix, the directivities of MFC and piezoelectric sheet in theoretical responses are plotted in Figures 9(a) and 9(b). As for the rectangular MFC and piezoelectric sheet, it is reported that their directivities are best when the wavelength is same with their length. As for the MFC directivity test, three different central frequencies are chosen for excitation tone burst. One of them coincides with the best directivity at which $\lambda=l$, namely, about $23 \mathrm{kHz}$, and the other two of them are less and more than $23 \mathrm{kHz}$, respectively, namely, $15 \mathrm{kHz}$ and $30 \mathrm{kHz}$. As for the directivity test of the piezoelectric sheet, two different central frequencies of the piezoelectric sheet are chosen. One of them coincides with the best directivity at which $\lambda=l$, namely, about $35 \mathrm{kHz}$, and the other is larger one about $40 \mathrm{kHz}$. Experimental directivities of MFC and piezoelectric sheet are plotted in Figure 9(c), where "PS" denotes the rectangular piezoelectric sheet.

It can be found that their directivities of MFC and piezoelectric sheet are not so well with the piezoelectric fiber, as shown in Figure 9. As for MFC, the response amplitude is decreasing as the angle $\theta$ increased from $0^{\circ}$ to 


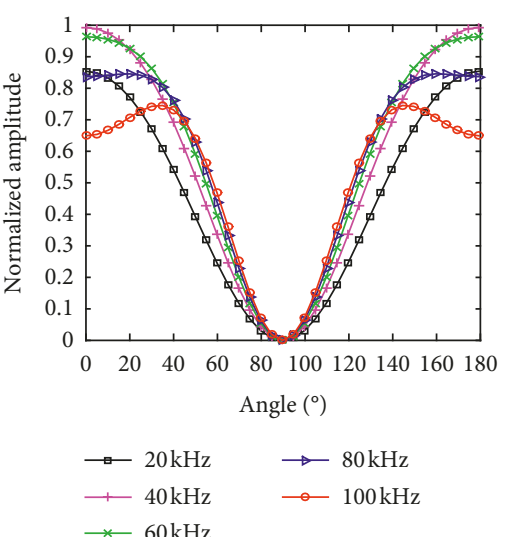

(a)

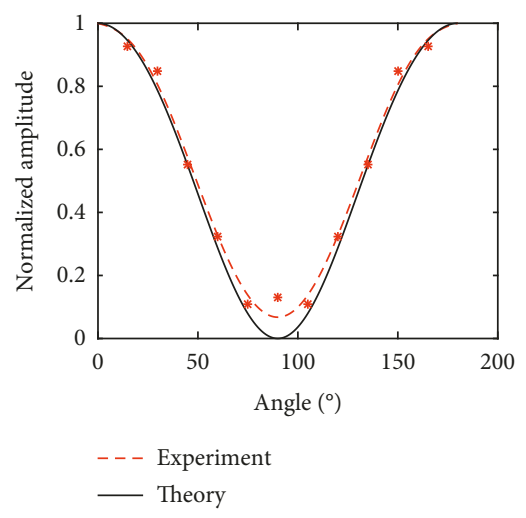

(b)

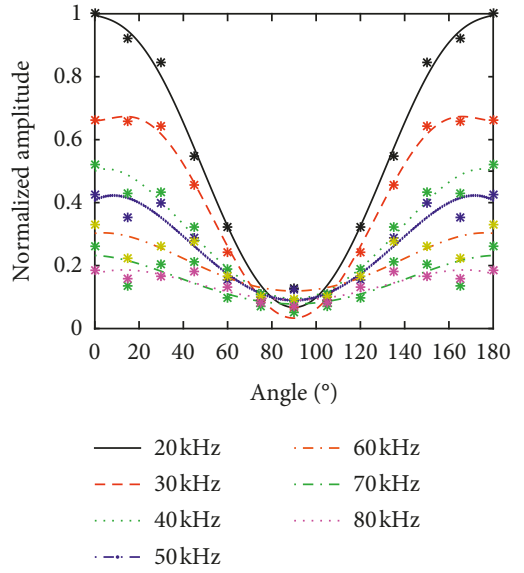

(c)

Figure 8: Theoretical and experimental directivity of the piezoelectric fiber: (a) theoretical directivity; (b) directivity at $20 \mathrm{kHz}$; (c) experimental directivity.

TABle 1: Wavelength of the flexural Lamb wave at different frequencies.

\begin{tabular}{lccccccc}
\hline $\begin{array}{l}\text { Frequency } \\
(\mathrm{kHz})\end{array}$ & 15 & 20 & 40 & 60 & 80 & 100 & 120 \\
\hline $\begin{array}{l}\text { Wavelength } \\
(\mathrm{mm})\end{array}$ & 30.78 & 26.57 & 18.58 & 15.01 & 12.86 & 11.38 & 10.28 \\
\hline
\end{tabular}

$90^{\circ}$ when $\lambda>l$; e.g., the wavelength is $30.78 \mathrm{~mm}$ at a frequency of $15 \mathrm{kHz}$. However, the response amplitude is increasing as the angle $\theta$ increased from $0^{\circ}$ to $90^{\circ}$ when $\lambda<l$, seen the approximate curve at the excitation frequency of $23 \mathrm{kHz}$ and $30 \mathrm{kHz}$. The curve trends of experimental results are in well agreement with the theoretical results. The large width and anisotropy material account for the complex directivity of MFC. Matt and Scalea [18] decomposed the directivity into a longitudinal and a transverse sensitivity factor to describe MFC's complex directivity. Because of the isotropy material of the rectangular piezoelectric sheet, the directivity performance is between MFC and piezoelectric fiber's performance. The response amplitude is increasing as $\theta$ increased from $0^{\circ}$ to $90^{\circ}$ when $\lambda>l$, namely, about $35 \mathrm{kHz}$. The response amplitude is also the combination of the longitudinal and the transverse sensitivity when $\lambda \leq l$, as well as the wavelength tuning effects. Compared with the rectangular MFC and piezoelectric sheet, it is found that the piezoelectric fiber shows better directivity than the MFC and piezoelectric sheet. The main reason is the smaller radius and the shorter length of the piezoelectric fiber.

3.3. Frequency Response Test Results. The predicted sensor responses are calculated from equations (15), (A.1), and (B.1). Theoretical frequency response spectrums of various piezoelectric sensors under different central frequencies are plotted in Figure 10(a), and all sensors are located in $0^{\circ}$, where "PF" denotes the piezoelectric fiber. The general behavior in Figure 10(a) is similar to what was found in [25]. The largest response in all cases is seen at low frequencies, and the tuning effects governed the responses at high frequencies because the wavelength has comparable magnitude with sensor's length. The response generally increases with decreasing sensor length, and thus, the piezoelectric fiber shows higher response than MFC and piezoelectric sheet in the low frequencies.

Considering the $A_{0}$ and $S_{0}$ motion waves are partially overlapping under the distance of $200 \mathrm{~mm}$ between the actuator and various sensors in the frequency range of $120 \mathrm{kHz}$ to $150 \mathrm{kHz}$, experimental tests are conducted in the low-frequency range from $20 \mathrm{kHz}$ to $120 \mathrm{kHz}$. Theoretical and experimental frequency responses of the piezoelectric fiber in different angels $\theta=0^{\circ}, 30^{\circ}$, and $45^{\circ}$ are plotted in Figures 10(b)-10(d). There is a reasonably good agreement between the experimental and theory curve. These differences could result from wave attenuation losses, measurement error, or the piezoelectric constant differences between the piezoelectric fibers.

Experimental response amplitudes of different piezoelectric sensors are presented in Table 2, where "PD" denotes the circular piezoelectric disc. The lengthwise directions of the tested piezoelectric fiber, piezoelectric sheet, and MFC are arranged in parallel with the Lamb wave propagation direction. It can be found that the piezoelectric fiber gives larger voltage amplitude than MFC, but less voltage than the piezoelectric disc in low-frequency below $40 \mathrm{kHz}$ while comparable voltage beyond $40 \mathrm{kHz}$. Piezoelectric sheet demonstrates the largest signal amplitude voltage $132.37 \mathrm{mV}$ at a low frequency of $20 \mathrm{kHz}$. Both piezoelectric sheet and piezoelectric disc give lower voltages at high frequencies. The response properties of the piezoelectric fiber are demonstrated to be capable of the sensoring Lamb wave propagation direction. The comparison results are significant to design or select the piezoelectric sensor in SHM applications. 


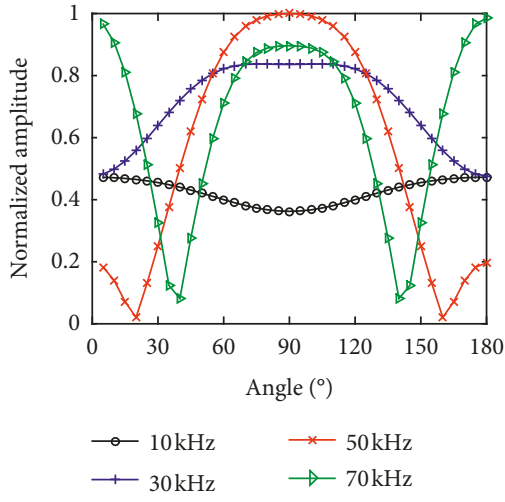

(a)

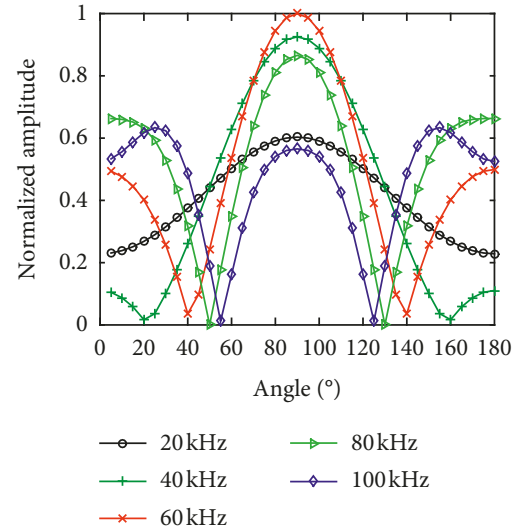

(b)

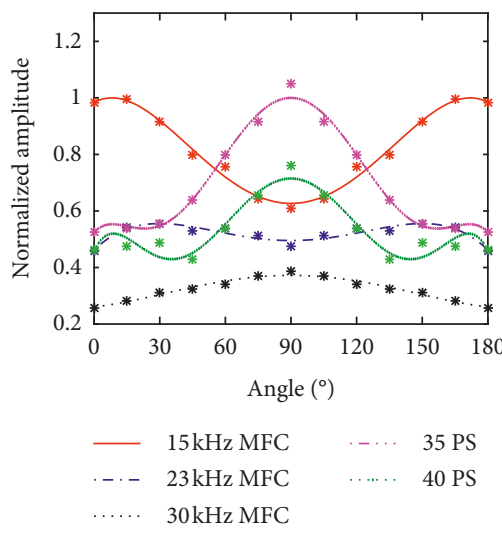

(c)

Figure 9: Theoretical and experimental directivity of MFC and piezoelectric sheet: (a) theoretical directivity of MFC; (b) theoretical directivity of the piezoelectric sheet; (c) experimental directivity.

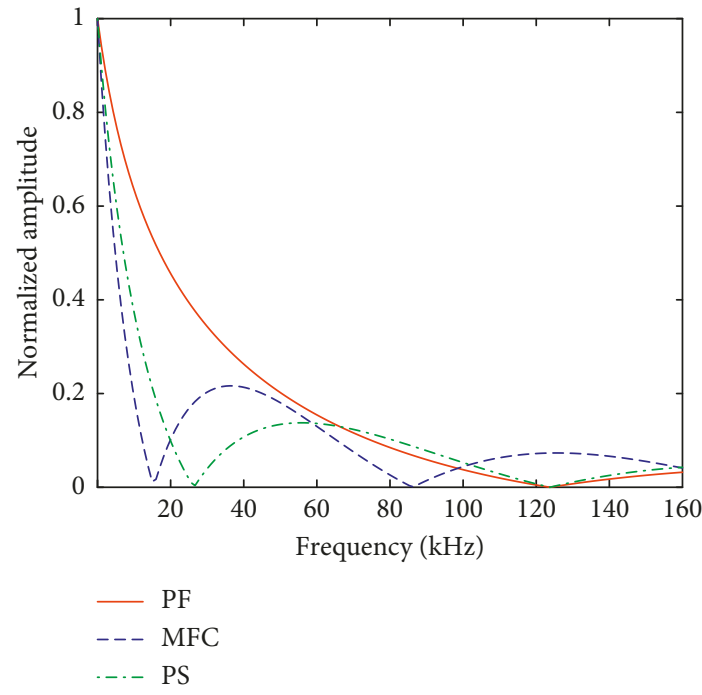

(a)

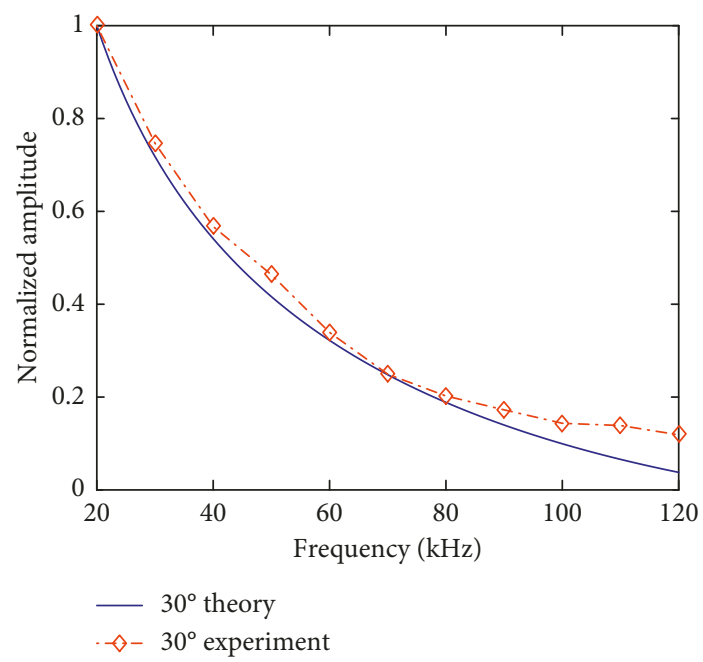

(c)

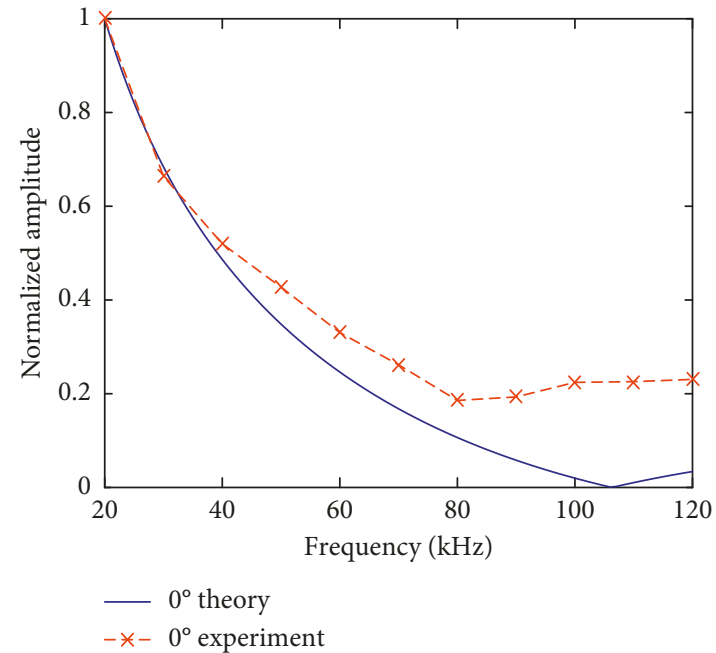

(b)

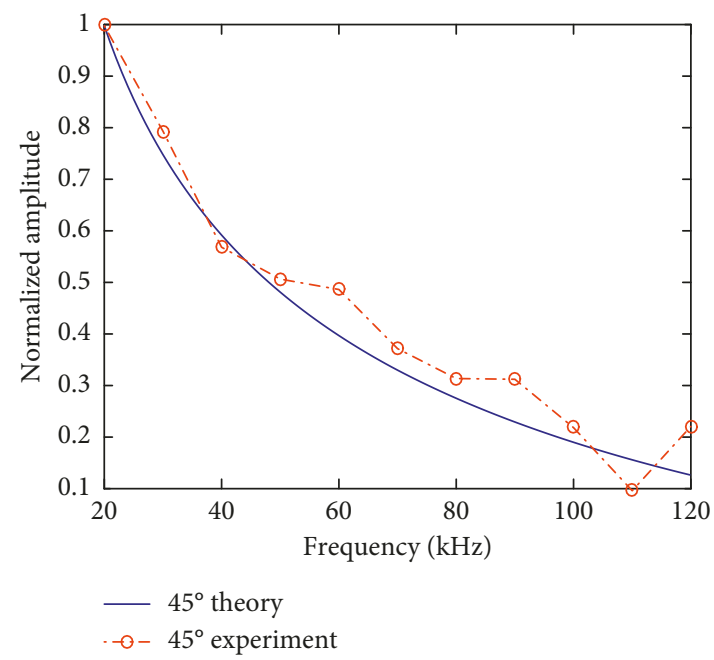

(d)

FIGURE 10: (a) Theoretical and experimental response amplitude spectra of the piezoelectric fiber at (b) $0^{\circ}$, (c) $30^{\circ}$, and (d) $45^{\circ}$. 
TABLE 2: Experimental maximum voltage amplitude of various piezoelectric sensors.

\begin{tabular}{|c|c|c|c|c|c|c|}
\hline Frequency & $20 \mathrm{kHz}$ & $40 \mathrm{kHz}$ & $60 \mathrm{kHz}$ & $80 \mathrm{kHz}$ & $100 \mathrm{kHz}$ & $120 \mathrm{kHz}$ \\
\hline Voltage of PF (mV) & 8.17 & 6.48 & 5.26 & 2.95 & 3.68 & 4.30 \\
\hline Voltage of MFC (mV) & 5.45 & 1.29 & 2.02 & 2.71 & 1.33 & 1.20 \\
\hline Voltage of PS (mV) & 132.73 & 63.86 & 23.67 & 30.73 & 21.87 & 4.70 \\
\hline Voltage of PD $(\mathrm{mV})$ & 36.30 & 32.00 & 9.87 & 4.72 & 4.16 & 3.83 \\
\hline
\end{tabular}

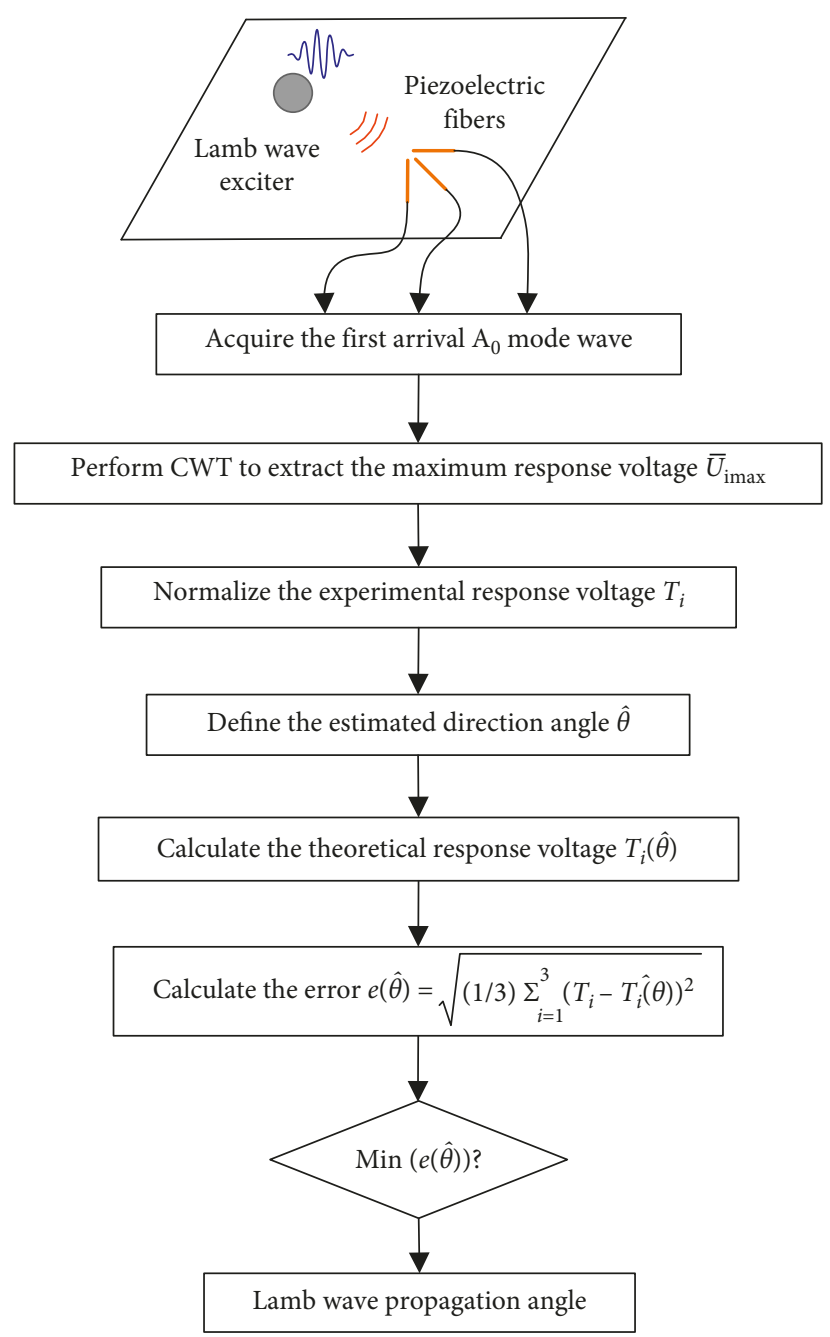

FIGURE 11: Block diagram of the proposed method.

3.4. Lamb Wave Propagation Direction Estimation Results. The above two experimental tests demonstrate that the piezoelectric fiber can be used as the Lamb wave directional sensor with good directivity and response characteristics. Two piezoelectric fiber rosettes are boned to the test specimen, and the actuator of a piezoelectric disc is located in the angle of $32^{\circ}$ and $55^{\circ}$. The distance between the actuator and the rosette is $277.2 \mathrm{~mm}$. The aluminum plate is the same with the above experimental test. The block diagram of the proposed method is shown in Figure 11. The error curve in a frequency of $70 \mathrm{kHz}$ is plotted in Figure 12(a), in which the angle step size in numerical computation is $0.05^{\circ}$, and the estimated angle results are plotted in Figure 12(b). It can be found that the accuracy of the estimated angles at different frequencies is mostly above $92 \%$. The errors are less at low frequencies below $40 \mathrm{kHz}$. The largest errors at certain frequencies are caused by the partially overlap of flexural and bending Lamb wave motion.

\section{Conclusion}

This paper proposed a directional sensor made of three piezoelectric fibers with a $0^{\circ} / 45^{\circ} / 90^{\circ}$ rosette configuration for sensoring the flexural Lamb wave direction. The directional response of the piezoelectric fiber is theoretically 


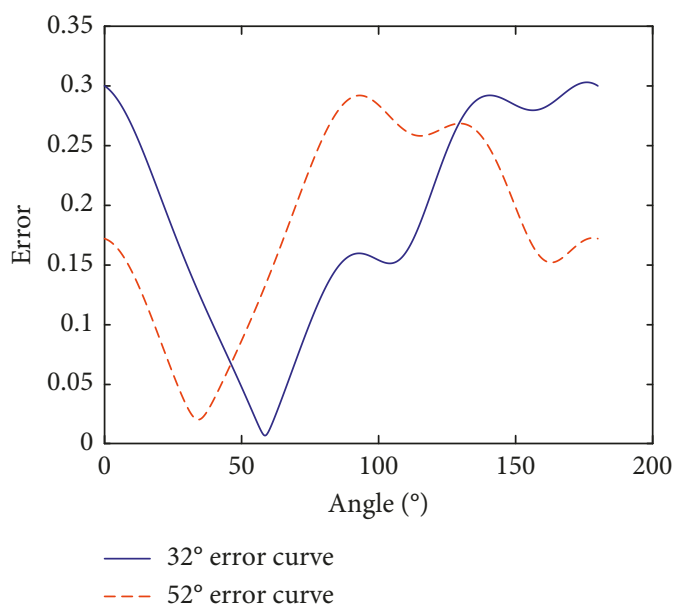

(a)

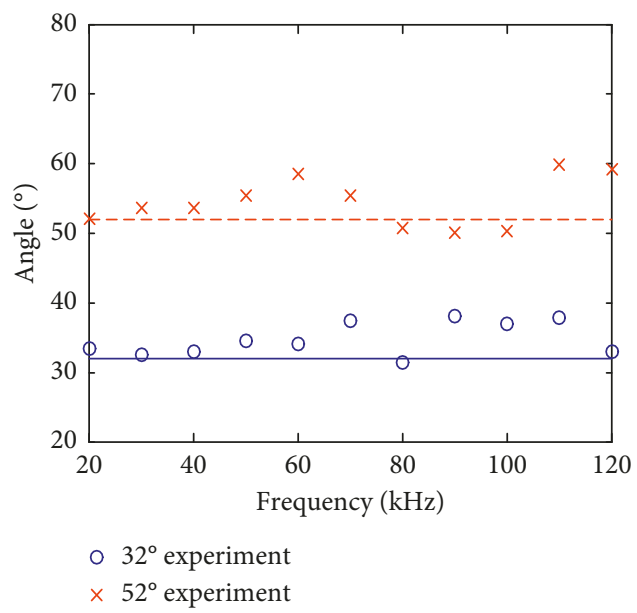

(b)

FIgURE 12: Test results of the Lamb wave propagation direction: (a) error curve in the central frequency of $60 \mathrm{kHz}$; (b) angle estimation results.

analyzed with narrowband tone-burst excitation. The directivity and the frequency response characteristics of piezoelectric fibers are demonstrated by comparing with other directional piezoelectric sensors, such as MFC, piezoelectric sheet, and piezoelectric disc. CWT is applied to extract the voltage response amplitude at central frequency from acquired Lamb wave signals. Theoretical and experimental results validate the capabilities of piezoelectric fibers to acquire the flexural Lamb wave propagation direction. The sum of the maximum response voltages of three piezoelectric fibers is used to normalize the response amplitude of each piezoelectric fiber, and a numerical estimation method using the error curve function is developed to determine the Lamb wave propagation direction. Experimental results validate the accuracy of the proposed method. In practical SHM applications, the damage-related Lame wave signal can be used to detect damage. The propagation direction of the scattered Lamb wave caused by damage can be determined with the proposed method. Future work will focus on damage localization by detecting the damage-related Lamb wave propagation direction with piezoelectric fiber sensors.

\section{Appendix}

The response equations of different piezoelectric sensors are as follows.

\section{A. MFC}

The response equations of MFC are as follows [18]:

$$
\bar{V}=\frac{4 N_{1} \lambda^{2} \bar{\varepsilon}_{x^{\prime} x^{\prime}}}{\pi^{2} l w \sin \theta \cos \theta} \sin \left(\frac{k w \sin \theta}{2}\right) \sin \left(\frac{k l \cos \theta}{2}\right),
$$

$$
\begin{aligned}
f(\theta) & =\frac{\left(A \cos ^{2} \theta+B \sin ^{2} \theta\right)}{\sin \theta \cos \theta} \sin \left(\frac{k w \sin \theta}{2}\right) \sin \left(\frac{k l \cos \theta}{2}\right) \\
A & =d_{31} E_{1}+d_{32} v_{12} E_{2} \\
B & =d_{31} v_{12} E_{2}+d_{32} E_{2}
\end{aligned}
$$

where $d_{31}$ and $d_{32}$ are the piezoelectric constants of the MFC. $E_{1}$ and $E_{2}$ are Young's modulus of the direction along the length and width, respectively. $\nu_{12}$ is Poisson's ratio.

\section{B. Rectangular Piezoelectric Element}

The response equations of the rectangular piezoelectric element are as follows [17]:

$$
\begin{aligned}
\bar{V} & =\frac{N_{2} \lambda^{2} \bar{\varepsilon}_{x^{\prime} x^{\prime}}}{\pi^{2} l w \sin \theta \cos \theta} \sin \left(\frac{k w \sin \theta}{2}\right) \sin \left(\frac{k l \cos \theta}{2}\right), \\
f(\theta) & =\frac{1}{\sin \theta \cos \theta} \sin \left(\frac{k w \sin \theta}{2}\right) \sin \left(\frac{k l \cos \theta}{2}\right) .
\end{aligned}
$$

\section{MPF}

The response equations of the MPF are as follows [24]:

$$
\begin{aligned}
\bar{V} & =\frac{2 N_{3} \lambda \bar{\varepsilon}_{x^{\prime} x^{\prime}}}{\pi e_{33}} \cos \theta \sin \left(\frac{\pi l \cos \theta}{\lambda}\right), \\
f(\theta) & =\cos \theta \sin \left(\frac{\pi l \cos \theta}{\lambda}\right) .
\end{aligned}
$$

\section{Data Availability}

The data used to support the findings of this study are available from the corresponding author upon request.

\section{Conflicts of Interest}

The authors declare that they have no conflicts of interest. 


\section{Acknowledgments}

The work of this paper was supported by the Hunan Provincial Key Research and Development Program (no. 2018GK2044) and the Hunan Provincial Natural Science Foundation (nos. 2017JJ2084 and 2018JJ4084).

\section{References}

[1] Z. Su, L. Ye, and Y. Lu, "Guided Lamb waves for identification of damage in composite structures: a review," Journal of Sound and Vibration, vol. 295, no. 3-5, pp. 753-780, 2006.

[2] V. Giurgiutiu, "SHM of aerospace composites-challenges and opportunities," in Proceedings of the CAMX Conference, Dallas, TX, USA, October 2015.

[3] Y. Bhuiyan, B. Lin, and V. Giurgiutiu, "Characterization of piezoelectric wafer active sensor for acoustic emission sensing," Ultrasonics, vol. 92, pp. 35-49, 2019.

[4] P. Kudela, M. Radzienski, and W. Ostachowicz, "Impact induced damage assessment by means of lamb wave image processing," Mechanical Systems and Signal Processing, vol. 102, pp. 23-36, 2018.

[5] J. Dai, X. Yang, and L. Wen, "Development of wind power industry in China: a comprehensive assessment," Renewable and Sustainable Energy Reviews, vol. 97, pp. 156-164, 2018.

[6] C. Q. Gómez Muñoz, F. P. García Marquez, B. Hernandez Crespo, and K. Makaya, "Structural health monitoring for delamination detection and location in wind turbine blades employing guided waves," Wind Energy, vol. 22, no. 5, pp. 698-711, 2019.

[7] E. Silva de Freitas and F. Guimarães Baptista, "Experimental analysis of the feasibility of low-cost piezoelectric diaphragms in impedance-based SHM applications," Sensors and Actuators A: Physical, vol. 238, pp. 220-228, 2016.

[8] T. Stepinski, M. Mańka, and A. Martowicz, "Interdigital lamb wave transducers for applications in structural health monitoring," NDT \& E International, vol. 86, pp. 199-210, 2017.

[9] X. Lin, G. Chen, J. Li, F. Lu, S. Huang, and X. Cheng, "Investigation of acoustic emission source localization performance on the plate structure using piezoelectric fiber composites," Sensors and Actuators A: Physical, vol. 282, pp. 9-16, 2018.

[10] X. Qing, W. Li, Y. Wang, and H. Sun, "Piezoelectric transducer-based structural health monitoring for aircraft applications," Sensors, vol. 19, no. 3, p. 545, 2019.

[11] A. A. Bent and N. W. Hagood, "Piezoelectric fiber composites with interdigitated electrodes," Journal of Intelligent Material Systems and Structures, vol. 8, no. 11, pp. 903-919, 1997.

[12] A. J. Brunner, M. Barbezat, C. Huber, and P. H. Flüeler, "The potential of active fiber composites made from piezoelectric fibers for actuating and sensing applications in structural health monitoring," Materials and Structures, vol. 38, no. 5, pp. 561-567, 2005.

[13] J. W. High and K. W. Wilkie, Method of Fabricating NASAStandard Macro-Fiber Composite Piezoelectric Actuator, NASA/TM-2003-212427 ARL-TR 2003-2833, Langley Research Center, Hampton, VA, USA, 2003.

[14] T. Kundu, H. Nakatani, and N. Takeda, "Acoustic source localization in anisotropic plates," Ultrasonics, vol. 52, no. 6 , pp. 740-746, 2012.

[15] D. C. Betz, G. Thursby, B. Culshaw et al., "Lamb wave detection and source location using fiber bragg gratin rosettes," Smart Structures and Materials 2003: Smart Sensor Technology and Measurement Systems, vol. 5050, pp. 117-128, 2003.
[16] G. Kawiecki and S. Jesse, "Rosette piezotransducers for damage detection," Smart Materials and Structures, vol. 11, no. 2, pp. 196-201, 2002.

[17] F. Lanza di Scalea, H. Matt, and I. Bartoli, "The response of rectangular piezoelectric sensors to Rayleigh and lamb ultrasonic waves," Journal of Acoustical Society of America, vol. 121, no. 1, pp. 175-187, 2007.

[18] H. M. Matt and F. L. D. Scalea, "Macro-fiber composite piezoelectric rosettes for acoustic source location in complex structures," Smart Materials and Structures, vol. 16, no. 4, pp. 1489-1499, 2007.

[19] S. Salamone, I. Bartoli, P. Di Leo et al., "High-velocity impact location on aircraft panels using macro-fiber composite piezoelectric rosettes," Journal of Intelligent Material Systems and Structures, vol. 21, no. 9, pp. 887-896, 2010.

[20] P. Kijanka, A. Manohar, F. Lanza di Scalea, and W. J. Staszewski, "Damage location by ultrasonic lamb waves and piezoelectric rosettes," Journal of Intelligent Material Systems and Structures, vol. 26, no. 12, pp. 1477-1490, 2015.

[21] M. Collet, M. Ruzzene, and K. Cunefare, "Generation of Lamb waves through surface mounted macro-fiber composite transducers," Smart Materials and Structures, vol. 20, no. 2, article 025020, 2011.

[22] M. Mańka, M. Rosiek, A. Martowicz et al., "Lamb wave transducers made of piezoelectric macro-fiber composite," Structural Control and Health Monitoring, vol. 20, no. 8, pp. 1138-1158, 2013.

[23] J. Liu, J. Qiu, W. Chang et al., "Lamb wave sensing with metalcore piezoelectric fiber for structural health monitoring," in Proceedings of the SPIE Smart Structures and Materials + Nondestructive Evaluation and Health Monitoring, vol. 7650, Article ID 765006, San Diego, CA, USA, March 2010.

[24] C. Zhang, J. Qiu, H. Ji, and S. Shan, “An imaging method for impact localization using metal-core piezoelectric fiber rosettes," Journal of Intelligent Material Systems and Structures, vol. 26, no. 16, pp. 2205-2215, 2015.

[25] V. Giurgiutiu, "Tuned lamb wave excitation and detection with piezoelectric wafer active sensors for structural health monitoring," Journal of Intelligent Material Systems and Structures, vol. 16, no. 4, pp. 291-305, 2005.

[26] B. Lin and V. Giurgiutiu, "Modeling and testing of PZT and PVDF piezoelectric wafer active sensors," Smart Materials and Structures, vol. 15, no. 4, pp. 1085-1093, 2006.

[27] J. Sirohi and I. Chopra, "Fundamental understanding of piezoelectric strain sensors," Journal of Intelligent Materials Systems and Structures, vol. 11, no. 4, pp. 246-257, 2000.

[28] M. Niethammer, L. J. Jacobs, J. Qu, and J. Jarzynski, “Timefrequency representations of Lamb waves," Journal of Acoustical Society of America, vol. 109, no. 5, pp. 1841-1847, 2001.

[29] J. Chen, J. Rostami, P. W. Tse, and X. Wan, "The design of a novel mother wavelet that is tailor-made for continuous wavelet transform in extracting defect-related features from reflected guided wave signals," Measurement, vol. 110, pp. 176-191, 2017.

[30] F. Li, G. Meng, K. Kageyama et al., "Optimal mother wavelet selection for lamb wave analyses," Journal of Intelligent Material Systems and Structures, vol. 20, no. 10, pp. 11471161, 2009. 


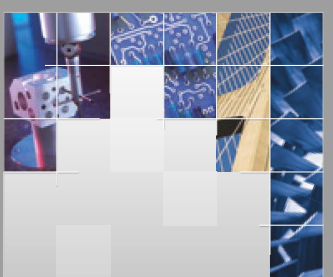

\section{Enfincering}
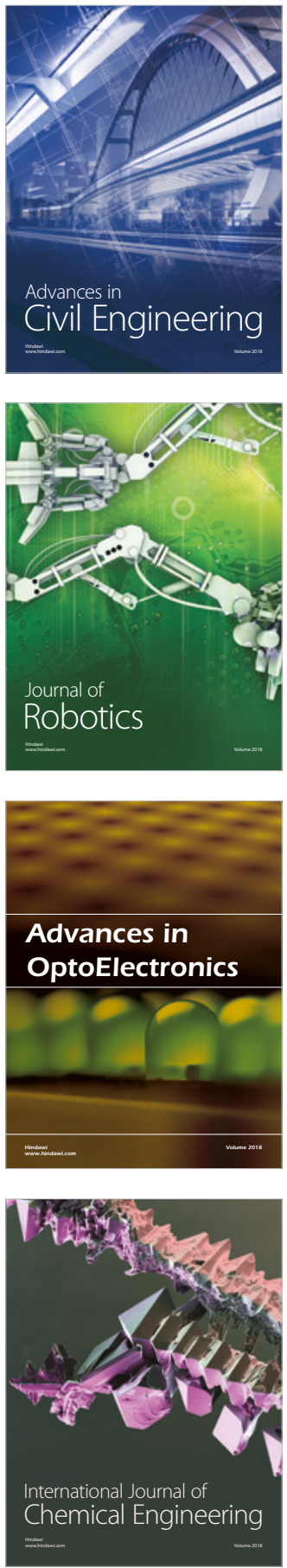

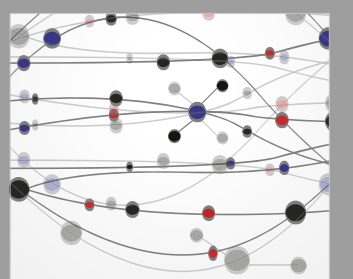

\section{Rotating \\ Machinery}

The Scientific World Journal

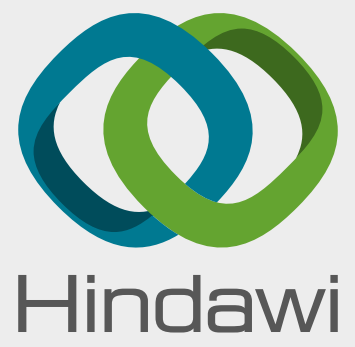

Submit your manuscripts at

www.hindawi.com
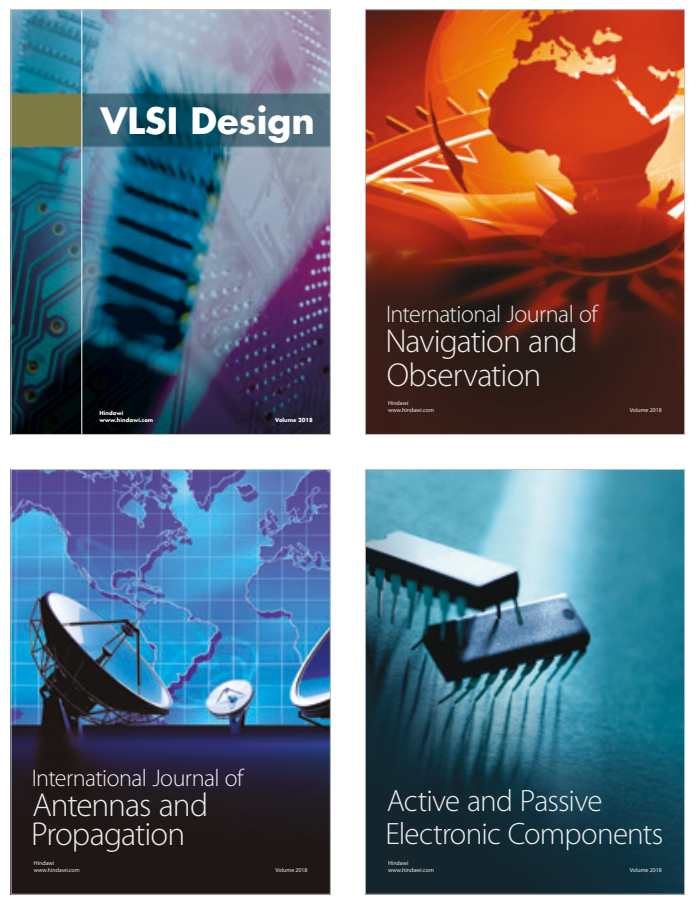
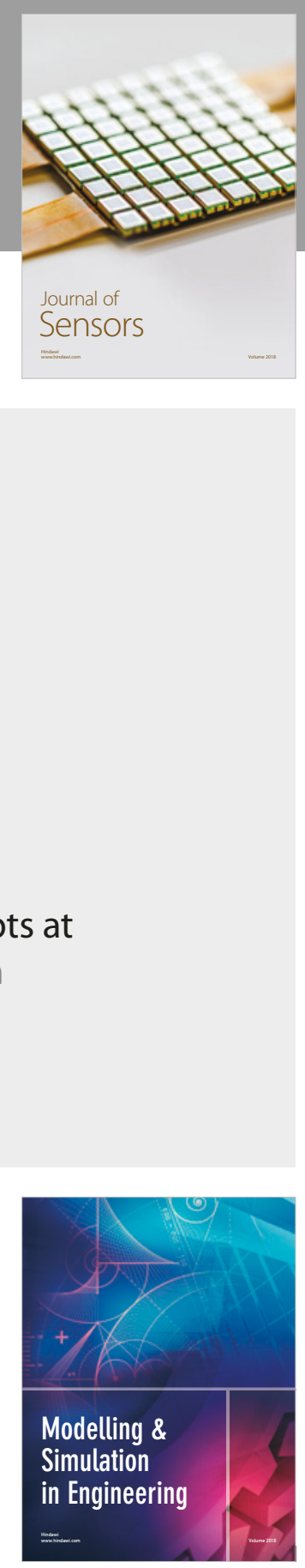

\section{Advances \\ Multimedia}
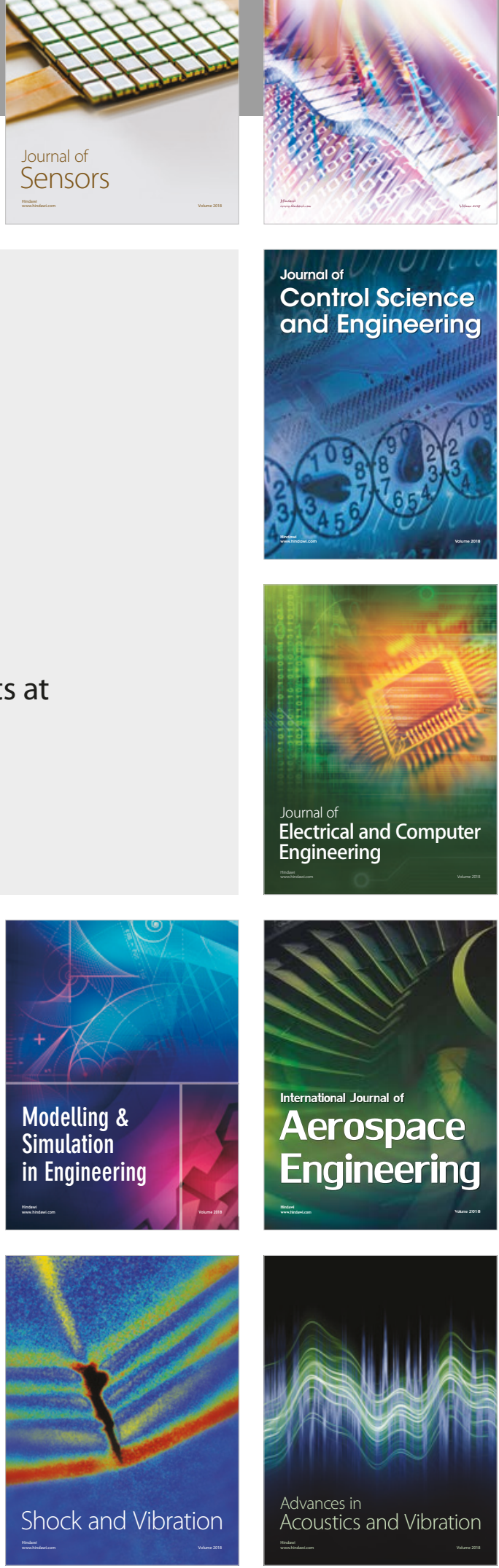